\title{
Wearable Smartphone: Wearable Hybrid Framework for Hand and Foot Gesture Interaction on Smartphone
}

\author{
Zhihan Lv \\ Norwegian University of Science and Technology, 7491 Trondheim, Norway \\ Institutionen för Tillämpad Fysik och Elektronik, Umeå University, 90187 Umeå, Sweden. \\ lvzhihan@gmail. com
}

\begin{abstract}
There is a increasing interest in creating wearable smartphone interaction technologies. A novel smartphone wearable hybrid interaction framework based on mixed low-cost hardware and software is proposed in this work. The software application renders touch-less application graphics and status information using smart phone's screen. Augmented reality image rendering technology is employed for a convenient hand/foot and phone interaction. The users interact with the application using hand/foot gesture motion behind the camera, which trigger the interaction event and generate activity sequences for interactive buffers. The touch-less algorithm provides a core support for hand/foot-gesture interaction by accurately detecting and tracking the hand/foot gesture. Four proof-of-concept application prototypes are developed based on the wearable hybrid framework. The user study evaluation demonstrates the social acceptability of the designed hand/foot gestures and the usability of the applications on proposed wearable hybrid framework with touch-less interaction approach.
\end{abstract}

\section{Introduction}

Many mobile graphic applications require touch and pointing input whether it is manipulating icons, menus and games in a mobile GUI. The problems with direct-touch finger input are 'the occlusion problem' and 'the fat finger problem' [29]. Touch-less interaction extends the operation space resolution at least 5-10 times higher than 2D touch for interacting with mobiles [30] and helps mitigate the problems of using mobile phone while user's hands are not available, for instance, their hands are dirty, or they are wearing gloves in winter, or they are driving and holding the steering wheel [3], so that improving safely in contexts where switching visual attention between the device and the physical environment pose safety concerns, because touchless interaction doesn't require the user to manipulate any

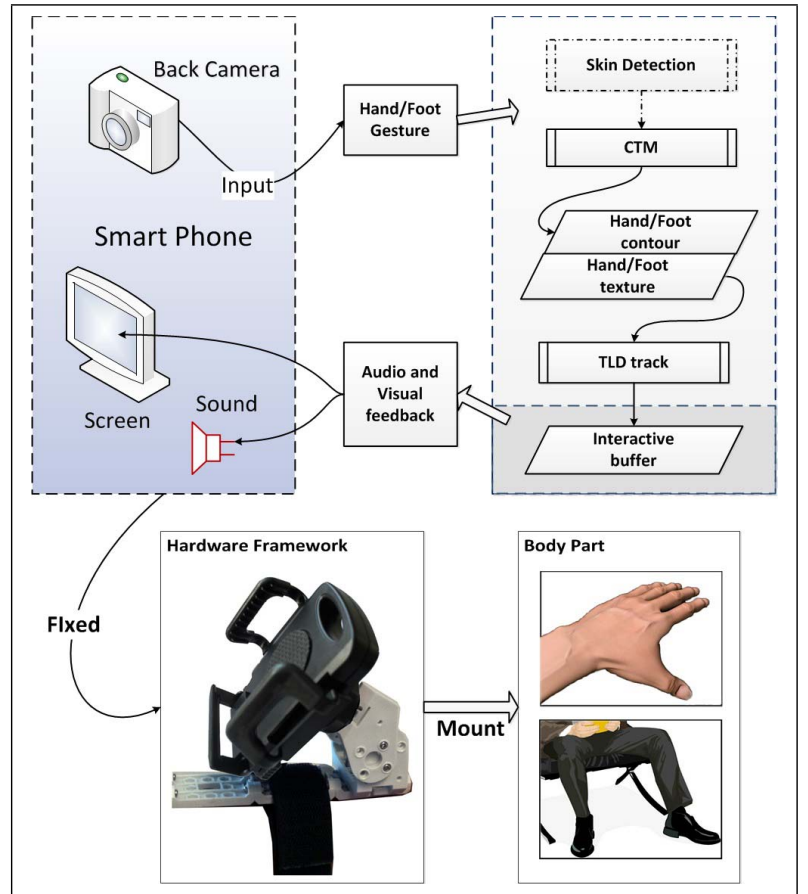

Figure 1. System Level Diagram depicting our hybrid framework flow chart.

more physical piece of hardware.

In addition, another motivation frequently mentioned is that touch-less interactions support more gestures in more natural patterns, which provide the less device-centric interaction techniques allowing a mobile user to focus attention on the task and its content instead of on the device while on the move. In this paper we explore and prototype a novel alternative touch-less approach to mobile interaction that uses a body-worn (i.e. wrist-worn and knee-worn) sensing system to capitalize on the real hand and foot the humans body provides. Early computer vision based wearable computing research projects, i.e. Gesture pendant [13], Wearable virtual tablet [26], Abracadabra [11], Gesture watch [15], 


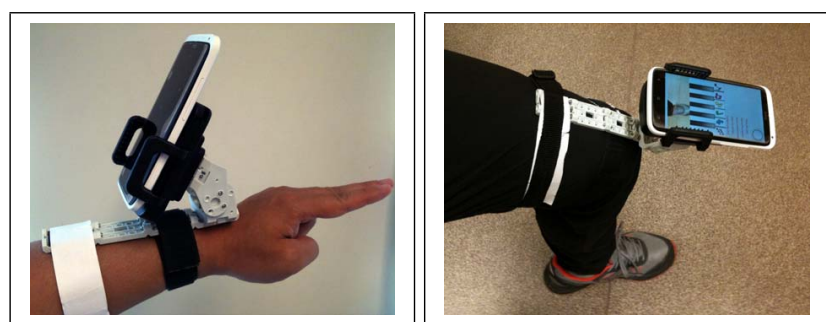

Figure 2. From left-to-right - The wearable framework mounts on wrist for hands (eg. finger) interaction. The wearable framework mounts on knee for feet interaction.

WUW [21], as well as dozens of ADI(Around Device Interaction) related technologies, i.e. OmniTouch [10], Ghost fingers [16], Markerless fingertip [4], Mouseless [20], Behand [6], Imaginary interfaces [8] inspires this hybrid wearable framework.

Recent research in mobile phone computing has led to a number of input techniques that allow for spatial input by hands beyond a touchscreen. Popular recent solution include: SideSight [5], Abracadabra [11], Minput [12] and SixthSense [19] all provide a level of spatial input in a mobile setting.

In addition to hands, we use feet along with hands in various everyday tasks (such as driving) and coordinated control mechanism of both modalities has been proven effectiveness in human computer interaction especially in virtual environments [27]. Considering handheld computing various foot gesture detection and tracking system have been proposed such as multitoe [2], kick [9], foot tapping [7] etc. Sangsuriyachot et al. [25] presented hand and foot gesture based interaction for tabletop environment. We believe that hand and foot gesture interaction can enhance immersive experience on handheld devices such as smart phone.

A parallel trend, camera phones are already popular and pervasive from 2006 [28], the miniaturization of mobile computing devices permit 'anywhere' access to the information [21], so the wearable device based camera initially present the wide applicable trend. An appealing feature of this kind wearable interfaces is that they could make it possible for users to communicate with computerized equipment without need for direct touch, and thus e.g. wearable device. The development of new touch-less interaction methods, supported largely by mobile smartphones and other wearable devices, have enabled an implicit loop of interaction between users and applications. Such interaction of wearable device using touch-less algorithm go beyond the traditional explicit graphical-based interfaces, can support novel and natural forms of interaction and include not only static gestures but also patterns of behaviour and even other dynamic physiological measures. Considering the current market trend and consumer demand on mobilephone interactions, it is challenging to sell the 'hand/foot touch-less on smartphone'idea when having another commercial component e.g., camera-mounted glasses, that isn't required to integrate with smartphone. However, the future mobile phone-like device had been envisioned as remaining a standalone device and vastly improved connectivity to other devices [1], while there is a increasing interest in creating wearable smartphone interaction technologies. By using the front or back camera of the smartphone mounted on wrist, knees or other parts of the body, the wearable smartphone sees what the user sees with the overlapped augmented object.

In this paper, we present a novel wearable bybrid interaction framework for smart phones where user mounts smart phone over wrist or knee and performs 'in air' interaction gestures. The primary contributions of this paper is a proposed software and hardware mixed hybrid platform for both hand and foot gesture interaction based on our ongoing work: an integrated real-time hand and foot detection and tracking algorithm based on the combination of Contour based Template Matching (CTM) complemented by skin detection and Tracking-Learning-Detection (TLD). Four applications on the hybrid framework are developmented as the demonstration of the concept and preliminary user study experiment with a two-stage questionnaire are designed to evaluate the social acceptability of the eleven designed hand/foot gestures and the usability of the four applications.

\section{Wearable Hybrid Framework}

The wearable hybrid framework integrate two part based on smartphone-centered design: (1) Wearable Hardware; (2) Touch-less Interaction algorithm. At run time, the hybrid framework fixes the smartphone on the forearm by a low-cost flexible wearable hardware and tracking the finger/foot motion (behind the screen) to simulate the touch event by a novel touch-less algorithm.

\subsection{Wearable Hardware}

The hardware of wearable hybrid framework is comprised of three main hardware components: mobilephone holder, holder base, fastener strip. The connection between mobilephone holder and holder base is a active loose-leaf, which can be twisted to adjust the pitch angle of the smartphone camera for adaptation of wrist and knee. The framework can be fixed on wrist or knee firmly by the fastener strip for detecting of hand and foot gesture interaction respectively. As in Figure 2. The cost of the hardware part, sans the smartphone, runs about $\$ 30$.

\subsection{Touch-less Interaction algorithm}

Our touch-less interaction algorithm uses dynamic programming based CTM algorithm [23] to localize the finger-contour for hand/foot, complemented optimally by 
lightweight skin detection [22], and employs TLD [14] framework to strengthen the tracking process in every frame. The touch-less interaction algorithm run in real-time which is especially targeted at mobile devices for the realization of the human-smartphone interaction on the hybrid framework, enabling a wide variety of gestures.

Our skin detection employs HSV color space of skin image to obtain the skin regions which are used to instantiate hypotheses for fingers in order to enable real-time hand segmentation even on resource-restraint devices.

CTM approach is an indispensable step for interactive hand (two fingers outstretched) detection in the beginning, because TLD cannot initialize by a generic hand/foot(shoe) model, moreover, the classification learning and training method cannot detect hands/feet which may not have distinctive feature. Hands template is modeled off-line in advance and then it is compared with image frames acquired from skin regions to find an optimal match. CTM allows a slight deformation of template, which makes it can detect successfully the approximate gesture of hands and feet of different people. CTM gives TLD a region of interest (ROI) for initialization (As in Figure 1).

TLD algorithm combines elements from tracking, learning and detection in the 2D image space to make it a longterm tracker. The TLD tracker uses a tracking strategy of the overlapping blocks, and tracks every block by LucasKanade optical flow method. TLD reduces the image processed time consuming of the pure CTM. Besides CTM exists a limitation of occasional false detection since the hand/foot gestures exceed the restriction of deformation or complex backgrounds interfere detection rate. The proposed combined touch-less algorithm successfully solves all of the unexpected detection results of hand/foot gestures. We already proved the high-success-rate of real-time detection and tracking for hand [17] and foot [18] by running test on large number of video captured on multi-context in our previous work. In this paper, we combined the algorithm into one core library with adaptation of the wearable hybrid framework, and adjust the parameters to enable detection and tracking for both hand and foot flexibly.

Our algorithm execution time is real time and accurate even when the hand/foot gesture is moving fast and in image blur cases. It's worth mentioning that the method can detect all kinds of the hand/foot gesture used in our application, as long as the correct hand/foot texture is detected in the first frame (As in Figure 3). The enhanced algorithm is followed as in Algorithm 1.

To improve the efficiency of the proposed algorithm, the size of first frame is scaled down to $25 \%$ for foot and $12.5 \%$ for hand and then CTM is applied. From the second frame the image is scaled to $12.5 \%$ for foot and $6.25 \%$ for hand, and then TLD tracking is employed (As in Figure 4). The scale ratio is different for hand or foot, due to the distance

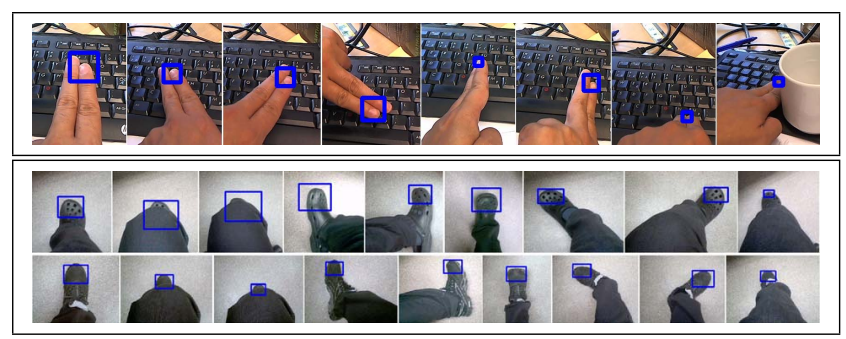

Figure 3. From up to down: The tracking results for a variety of finger gestures with background interference. The tracking results for a variety of foot-gestures with either obvious (1st row) or weak features (2nd row).

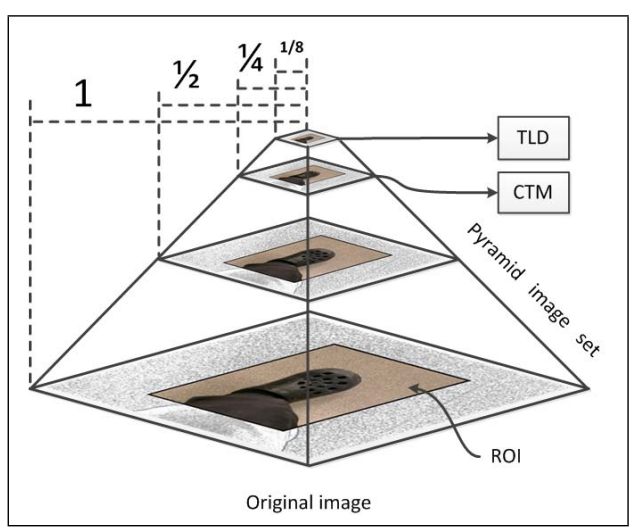

Figure 4. Pyramid structure for accurate and real time results.

between the camera of the smartphone with hand is closer than foot (As in Figure 2). Both the 'hand target test' and 'foot target test' results indicate both efficiency and success rate of the tracking is close to $100 \%$.

To evaluate our system, we have developed a series of proof of concept apps. The apps are respectively 'Social media site launch' and 'Bouncing ball game' for hand gesture touch-less, as well as 'Football game' and 'Foot-Play Piano' for foot gesture touch-less. In the 'Social media site launch' app users are asked to perform touch-less clicks and launch desired social media site (as shown in Figure 5). It demonstrates easy-to-use and accurate touch-less interactions. 'Bouncing ball game' and 'Football game' have the similiar game rules beside active controlling body are respectively hand and foot. 'Foot-Play Piano' proves the capability of touche-less to control rhythm by foot.

\section{Wearable Smartphone: System and Appli- cation}

We present four proof-of-concept wearable implementations based on wearable hybrid system prototype that demonstrate the usefulness, viability and flexibility of the framework, in order to hereafter investigate its possibilities and limitations and to illustrate and explore different use 


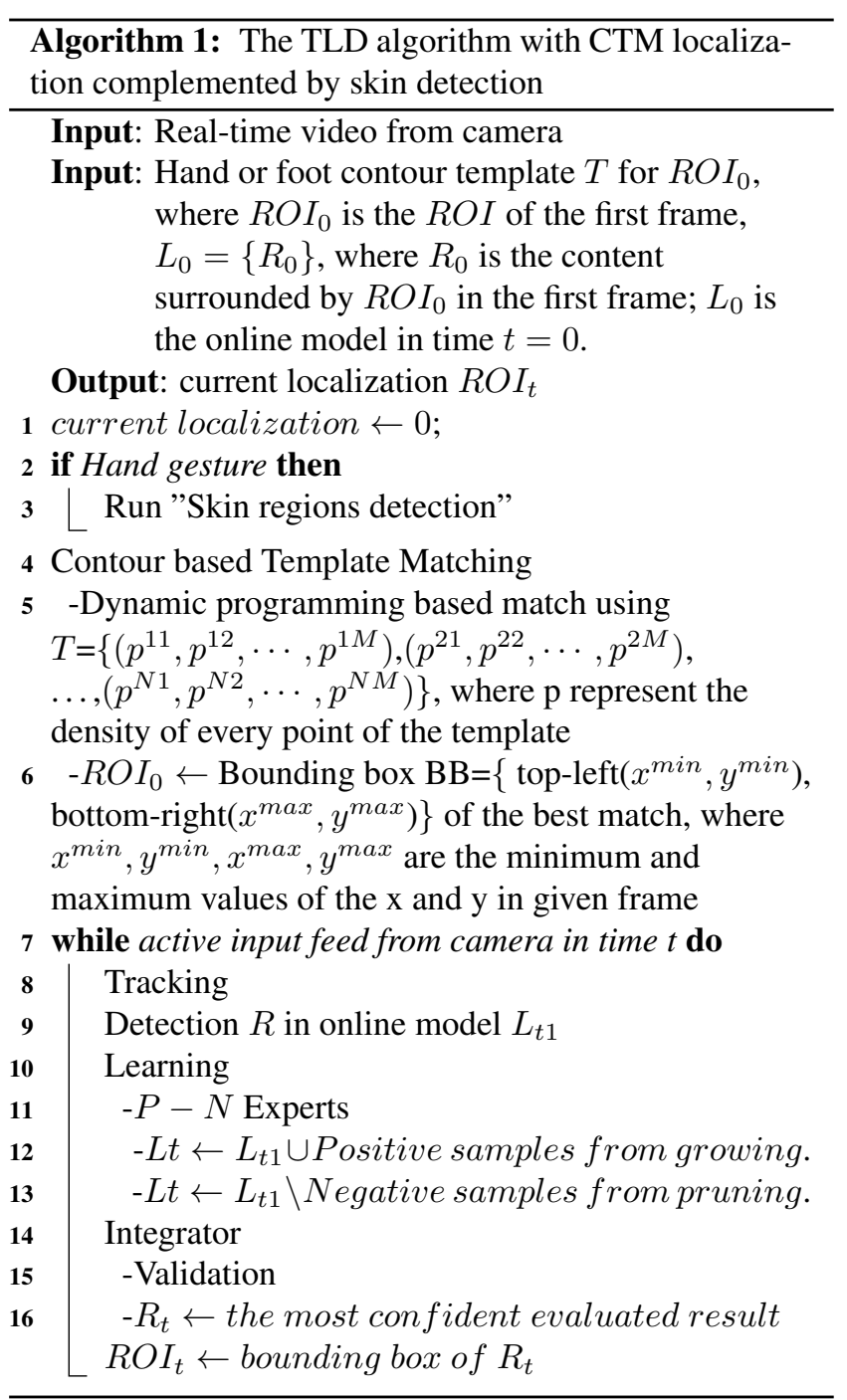

cases. And then we report on a user study comparing social acceptability and usability.

The final wearable software system prototype was built using three-layers augmented interaction GUI. The function of bottom layer is rendering the background and scenario of the application. The middle layer performs mainly user finger gesture by real-time video of camera view superposed over the bottom layer. The top layer render the augmented object and the overlay information of the application scenarios such as menu, icon, game boundary. The augmented rendered glove/shoes image or color-marked cursor overlay onto the camera view on the screen provides the user with direct and immediate reference of the actual hand position relative to the screen. We expect the user to perceive the touch-less approach as being stretched from his/her body (i.e. hand, foot) for manipulating content on the screen. In order to complete this interaction loop, our system maps the physical hand/foot onto the screen coordinates. When the

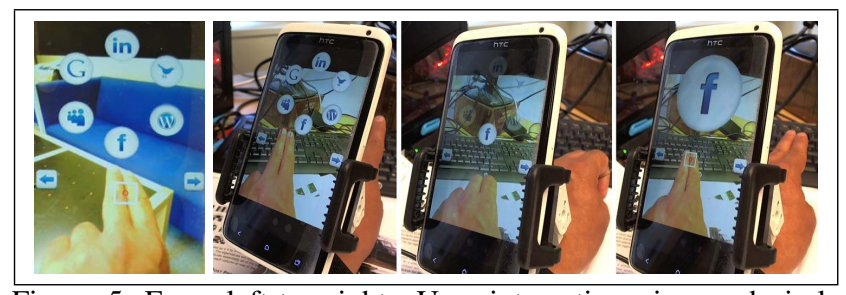

Figure 5. From left-to- right: User interaction view and circle menu application scenarios with the responds to 'Finger Flexion and Extension' gesture.

user manipulate the icon/button on the screen by touch-less gestures, the system updates the position of the augmented rendered image/cursor overlapping with the screen mapped from the location of the gesture accordingly. Touch-less algorithm recognizes a number of user gestures ranging from the simple action of 'Swing Finger' to more complex operations such as 'Finger Flexion and Extension'. The mobile phone is mounted on the wrist by a simple flexible plastic stand. All applications were implemented in $\mathrm{C}++$, Java with Android SDK/NDK and the image processing tasks were realized using OpenCV. Our test device was an HTC one X phone equipped with a $1 \mathrm{GHz}$ processor, $1 \mathrm{~GB}$ RAM, and a 8 megapixel camera and running Android 4.1.

\subsection{Social media site launch}

Based on the wearable method, we have developed a 'social media site launch' application: ie., 'Wearable Circle Menu' on Android platform. Wearable Circle Menu is a finger gesture based round-shape menu which can lunch several popular social media sites. Users rotate the suspended circle menu and select the augmented icon using finger gesture from the mobile phone's full-screen camera view. To access the required icon, one can use 'Swing Finger' gesture to make the circle menu rotating and select any icon on the mobile display, ie. 'Swing Finger Left' gesture to rotate clockwise 'Swing Finger Right' means anticlockwise, until the expected icon is on the bottom of the circle menu. The expected icon can be selected by Finger Flexion and Extension' gesture, and unselected by the same gesture. As in Figure 5.

\subsection{Bouncing ball game}

A bouncing ball game application based on our touchless interaction approach is developed on the Android platform as a proof of the supporting of speed and orientation based movement gesture of touch-less concept. The scene of the game is a handball court with a camera view overlay. An augmented reality glove image is rendered following the finger gesture. The handball always bounces back and forth on the court area until it's into the goal or the player uses the gesture 'Collide the ball' to intercept and hold it. When the player holds the ball, s/he can use the gesture 'Swing 

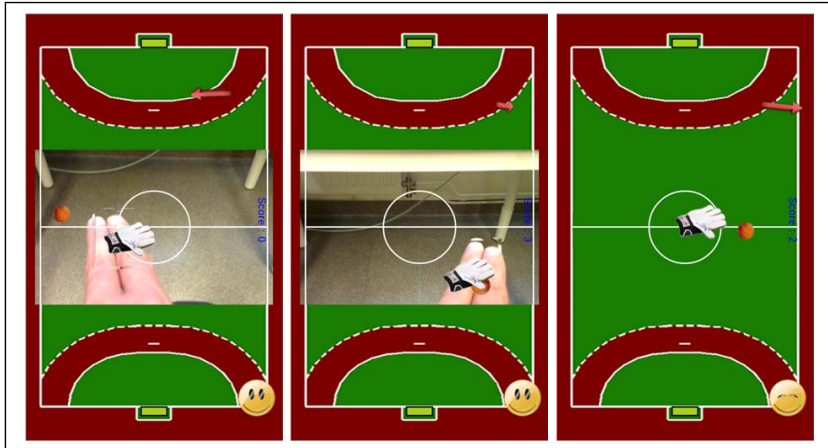

Figure 6. From left-to- right: Intercept /Dribble gesture and hidden camera view of the game UI.

Finger Slowly' to dribble the ball to the desired position. 'Swing Finger Fast' gesture can be used for throwing or bunting the ball, at the same time a red arrow at the topright corner of the screen is the index of the speed value by degree of elongation. In order to compare the effects of the augmented reality rendering interaction UI and pure graphic UI, a 'smile face' button at the right-bottom of the screen is developed to trigger hide/show status of the camera view. As in Figure 6.

\subsection{Football game}

We develop a real-time multimodal football game smart phone application. It renders the game graphics and game status information using smart phone's screen, audio and vibration. Augmented reality image rendering technology is employed. The players interact with the game using foot gestures, which triggers the interaction event and generate activity sequences for interactive buffers. The trajectory of the football is changed by interactive buffers. In the system, the players use real foot to kick the ball using 'Kick The Ball' on the smart phone screen. When the player holds the ball, s/he can use the gesture 'Move Foot Slowly' to dribble the ball to the desired position. 'Move Foot Fast' gesture can be used for throwing or bunting the ball. As in Figure 7 left.

The Bouncing ball game and the Football game have the same game rules for hand and foot interaction respectively, which are as following:

1. The player interacting-hand/foot must be placed in mobile camera view for $5 \mathrm{sec}$. for detection and localization.

2. The ball always bounces back and forth on the whole screen area till end of the game and the players try to keep the ball (using hand to touch or foot to kick) under his/her control.

3. The player get 1 score if she/he touches (or kicks) the ball once, and the game is over if the ball enters into the highlighted goalpost.

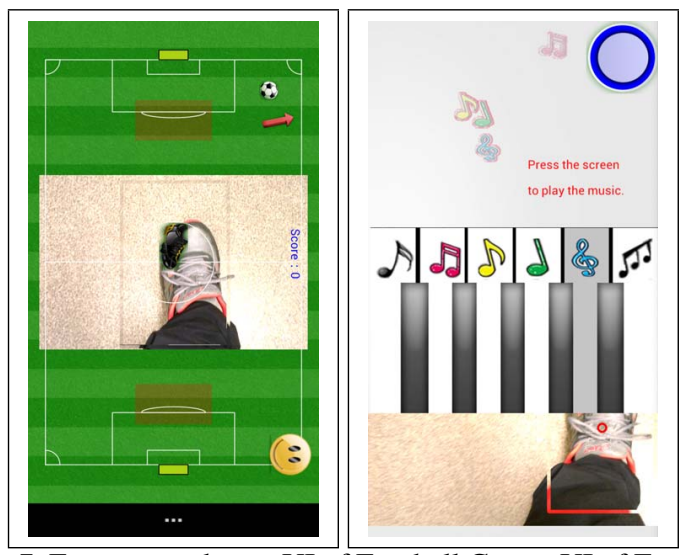

Figure 7. From up to down: UI of Football Game. UI of Foot-Play Piano App

\subsection{Foot-Play Piano}

The user study application scenario namely Foot-Play Piano is developed to demonstrate the rhythm control of touch-less interaction. This application gives an option to play a music on the users android based smart phone using foot-gesture. There is a circle progress plate on the righttop coner of the screen, which is used to show how long the user press the piano key, eg. a circle is one mora, semicircle is a half mora. When the volume up button is pressed by 'Press The Key' gesture, the application starts to look for the movement of the foot through android camera. When a collision is detected with any of the augmented piano-key, the code related to that key is generated by the program, and the circle progress plate whirls until the user release the piano key by 'Release The Key' gesture. After the press of every note of the music, the users can play the music at once. This application works on the above mentioned three layer structure. The first layer displays augmented graphics where each piano-button has an ascribed specific code. The second layer is responsible for communication between upper and lower layer. As in Figure 7 right.

\section{Preliminary User Study}

In order to evaluate the social acceptability of the applications and the usability of the gestures, 15 participants (6 female), from a diverse background (9 students from different disciplines: computer science (5), electron(1), biol$\operatorname{ogy}(1)$, art (1), and law (1), 6 working professionals from different industries: education (3), banking (1), policeman (1), and market (1)) aged from 25-46 $(\mathrm{m}=30.13$, $\mathrm{sd}=5.74)$, were given the opportunity to interact with the applications, and were asked targeted and open-ended questions. None had experience with touch-less interfaces on smartphone. First of all, the moderator introduced the concept of 'wearable' with the demonstration. We designed a two-stages 


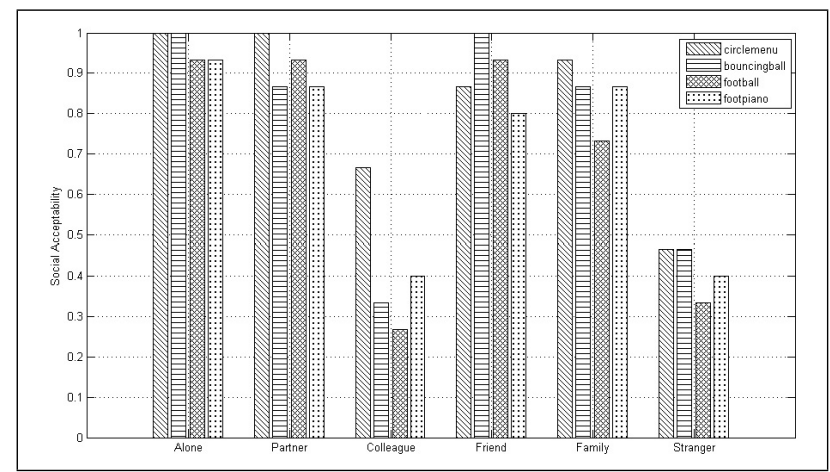

Figure 8 . Social acceptability rating by audiences and applications.

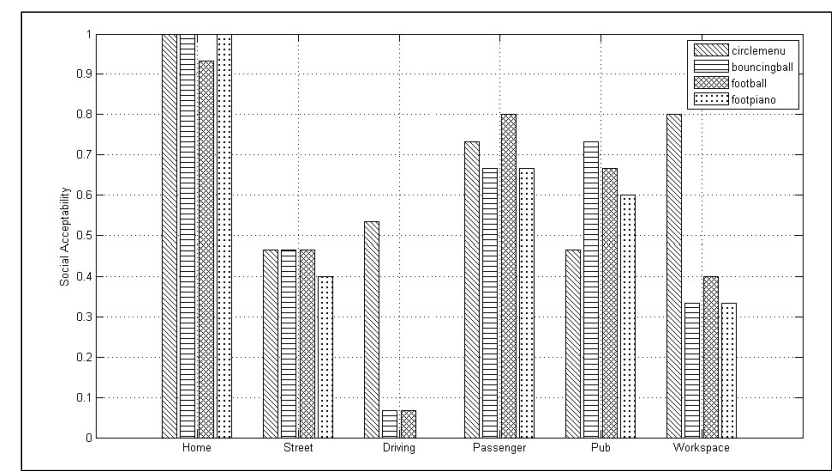

Figure 9. Social acceptability rating by locations and applications. questionnaire (and the design of the experiment) to elicit participants' reactions to this modality.

The first stage is comprised of the same questions as that inspired by Rico et al. [24], meant to elicit social acceptability of both applications. Rico et al. distinguish social acceptability by two factors: audience (alone, partner, colleague, friend, family, stranger) and location (home, passenger, workspace, pub, street, driving). The second stage is comprised of Likert scale questionnaire to reveal the usability of the eleven designed gestures including six hand touch-less gestures ('Swing Finger Left', 'Swing Finger Right', 'Finger Flexion and Extension', 'Swing Finger Slowly', 'Swing Finger Fast', 'Collide the ball') in the two applications based on hand interaction, and five designed gestures ('Kick The Ball', 'Move Foot Slowly', 'Move Foot Fast', 'Press The Key', 'Release The Key') in the two applications based on foot touch-less interaction. It comes as no surprise that these interaction metaphors found their way into modern body-interaction-enabled applications on devices ranging from smart phones and tablets to large tabletop displays. We think these metaphor will be well established for wearable hybrid framework based on touch-less interaction.

To evaluate the social acceptability of the applications, the participants watched a video showing a tutorial performing each gesture of both applications. Then, the first stage questionnaire was administered and participants were invited to try the device in person. In the second stage, a smartphone was mounted onto the wrist and then knee of each participant and performed the four applications. Afterwards the second stage questionnaire was administered. Measurements came in the form of both questionnaires.

The mean acceptance rating across all contexts is above 0.5 for each application ( $m_{\text {circlemenu }}=$ $0.7444 ; m_{\text {bouncingball }}=0.6500 ; m_{\text {football }}=$ $\left.0.6222 ; m_{\text {footpiano }}=0.6056\right)$. These results are illustrated in Figure 8 and Figure 9. The Kruskal-Wallis $(\mathrm{KW})$ test $\left(\chi^{2}=9.19, p=0.0269\right)$ reveals a significant effect on social acceptability for the applications at $p=.05$ level., followed by pair-wise comparisons reveal that 'circlemenu' is more acceptable than 'bouncingball' and 'football' is a bit more acceptable than 'footpiano'. 'circlemenu' is the most popular among the four apps. (meanrank $_{\text {circlemenu }}=392.5$, meanrank $k_{\text {bouncingball }}=$ 358.5, meanrank $k_{\text {football }}=348.5$, meanrank $_{\text {footpiano }}=$ 342.5). It was noted that participants inclined to use 'circlemenu' in work or attention paid context (colleague, workspace, driving), and play 'bouncingball', 'football', 'footpiano' in open context (pub). Moreover, the social acceptability reveals that the entertainment applications are unwelcome in work context (colleague, workplace).

The mean usability rating indicate that all gesture are applicable. The standard deviation of the usability of the 'Swing Finger Slowly' gesture is much larger than others and indicates that there was less consensus among participants about the usability of this gesture in Table 1.

The KW test reveals a significant effect $\left(\chi^{2}=\right.$ $24.63, p=0.0061)$ on usability for the gestures at $p=.01$ level. The mean-rank followed reveals that gesture 'Finger Flexion and Extension' get the highest score and 'Swing Finger Slowly' get the lowest score for the usability.

Table 1. Mean and Standard Deviation of usability of gestures

\begin{tabular}{|c|c|c|c|c|c|c|}
\hline & $\begin{array}{c}\text { Swing } \\
\text { Finger Left }\end{array}$ & $\begin{array}{l}\text { Swing } \\
\text { Finger } \\
\text { Right }\end{array}$ & $\begin{array}{l}\text { Finger Flexion } \\
\text { and Extension }\end{array}$ & $\begin{array}{l}\text { Swing } \\
\text { Finger } \\
\text { Slowly }\end{array}$ & $\begin{array}{c}\text { Swing } \\
\text { Finger Fast }\end{array}$ & $\begin{array}{l}\text { Collide the } \\
\text { ball }\end{array}$ \\
\hline $\mathrm{m}$ & 4.2000 & 4.0667 & 4.6000 & 3.4000 & 4.1333 & 4.2000 \\
\hline \multirow[t]{2}{*}{ sd } & 0.7746 & 0.7988 & 0.5071 & 1.1212 & 0.9155 & 0.7746 \\
\hline & Kick The Ball & & \multicolumn{2}{|c|}{ Move Foot Fast } & $\begin{array}{l}\text { Press The } \\
\text { Key }\end{array}$ & $\begin{array}{l}\text { Release The } \\
\text { Key }\end{array}$ \\
\hline $\mathrm{m}$ & 3.4667 & \multirow{2}{*}{$\begin{array}{l}3.5333 \\
0.9904\end{array}$} & \multicolumn{2}{|c|}{3.6667} & 4.0667 & 3.7333 \\
\hline sd & 0.6399 & & \multicolumn{2}{|c|}{1.0465} & 0.7988 & 1.0328 \\
\hline
\end{tabular}

In addition, each participant answers two extra questions: Do you prefer the visual camera view shown in the application 'bouncing ball game'? Is the wearable hardware stand ok? The result indicates that the augmented reality camera view is an expected UI component ( $m=$ $0.8667, s d=0.7432)$ and the simple extra hardware stand is acceptable by most participants $(m=0.9333, s d=$ 0.2582 ). In this extra study we could indentify that ap- 
proximately $93 \%$ of the respondents are willing to mount the extra hardware on their wrist or knee to interact with their hand or foot for simple use case. This reflects a high potential for the wearable hybrid framework based on touch-less algorithm, which is leveraged by extra-hardwaredependence as the main drawbacks of this interaction style.

\section{Conclusions}

In this paper, we give a brief overview of prior research relevant to wearable smartphone technology. Proposed wearable hybrid framework allows user to perform touch-less interaction when their hand are covered and/or busy, and gives a full preview of hand or foot not just the hover and touch points. Current prototype system, although fun to use, have a few limitations, for example, for hand it can only perform side-to-side two fixed fingers gesture, normal full hand gestures are not considered in first version. It is also unable to detect rotation gestures. In future studies, we will carefully consider hardware and software modification to extract the hand/foot gesture orientation (i.e., yaw, pith, and roll) parameters, and plan to address these limitations in the future prototypes.

Concerning the use cases, we completed the presented four application prototypes in order to conduct early user study comparing these novel interaction styles with established ones and identify the most promising applications from the wide range of novel interaction possibilities. User studies confirm that the wearable hybrid framework are intuitive and controllable and that mappings between gesture and manipulate on mobile phone.

Our solution provides a low-cost solution for the professional application on mobile phone, even the future wearable device such as projected glasses, Memoto or GoPro. The wearable hybrid framework capability demonstrated in this paper is also potentially applicable to wear on other body parts. In addition, it is also possible to replace it with other types of sensors to improve the touch-less interaction modality of the hybrid framework. This may include capacitive, electronic-field, ultrasound, or IR-based sensors, which may be capable of generating a hand/foot image from the raw data. We believe that it's entirely possible to perform multi-modal tasks and communications using wearable hybrid framework based smart phone's interaction.

\section{Acknowledgments.}

The author is thankful to the colleagues at Umeå university and KTH in Sweden for the successful cooperation.

\section{References}

[1] O. Amft and P. Lukowicz. From backpacks to smartphones: Past, present, and future of wearable computers. IEEE Pervasive Computing, 8(3):8-13, 2009.
[2] T. Augsten, K. Kaefer, R. Meusel, C. Fetzer, D. Kanitz, T. Stoff, T. Becker, C. Holz, and P. Baudisch. Multitoe: high-precision interaction with back-projected floors based on high-resolution multi-touch input. In 23rd Annual ACM Sym. UIST, 2010.

[3] G. Bailly, J. Müller, M. Rohs, D. Wigdor, and S. Kratz. Shoesense: a new perspective on gestural interaction and wearable applications. In Proceedings of the SIGCHI Conference on Human Factors in Computing Systems, CHI '12, pages 1239-1248, New York, NY, USA, 2012. ACM.

[4] M. Baldauf, S. Zambanini, P. Fröhlich, and P. Reichl. Markerless visual fingertip detection for natural mobile device interaction. In Proceedings of the 13th International Conference on Human Computer Interaction with Mobile Devices and Services, MobileHCI '11, pages 539-544, New York, NY, USA, 2011. ACM.

[5] A. Butler, S. Izadi, and S. Hodges. Sidesight: multi-"touch" interaction around small devices. In Proceedings of the 21 st annual ACM symposium on User interface software and technology, UIST '08, pages 201-204, New York, NY, USA, 2008. ACM.

[6] M. L. Caballero, T.-R. Chang, M. Menéndez, and V. Occhialini. Behand: augmented virtuality gestural interaction for mobile phones. In Proceedings of the 12th international conference on Human computer interaction with mobile devices and services, MobileHCI '10, pages 451-454, New York, NY, USA, 2010. ACM.

[7] A. Crossan, S. Brewster, and A. Ng. Foot Tapping for Mobile Interaction. In 24th BCS Conf. HCI, UK, 2010.

[8] S. Gustafson, D. Bierwirth, and P. Baudisch. Imaginary interfaces: spatial interaction with empty hands and without visual feedback. In Proceedings of the 23nd annual ACM symposium on User interface software and technology, UIST '10, pages 3-12, New York, NY, USA, 2010. ACM.

[9] T. Han, J. Alexander, A. Karnik, P. Irani, and S. Subramanian. Kick: Investigating the Use of Kick Gestures for Mobile Interactions. In 13th Int. Conf. HCI with Mobile Devices and Services, 2011.

[10] C. Harrison, H. Benko, and A. D. Wilson. Omnitouch: wearable multitouch interaction everywhere. In Proceedings of the 24th annual ACM symposium on User interface software and technology, UIST '11, pages 441-450, New York, NY, USA, 2011. ACM.

[11] C. Harrison and S. E. Hudson. Abracadabra: wireless, highprecision, and unpowered finger input for very small mobile devices. In Proceedings of the 22nd annual ACM symposium on User interface software and technology, UIST '09, pages 121-124, New York, NY, USA, 2009. ACM.

[12] C. Harrison and S. E. Hudson. Minput: enabling interaction on small mobile devices with high-precision, low-cost, multipoint optical tracking. In Proceedings of the SIGCHI Conference on Human Factors in Computing Systems, CHI '10, pages 1661-1664, New York, NY, USA, 2010. ACM.

[13] T. S. Jake, J. Auxier, and D. Ashbrook. The gesture pendant: A self-illuminating, wearable, infrared computer vision system for home automation control and medical monitoring. In In International Symposium on Wearable Computing, pages 87-94, 2000. 
[14] Z. Kalal, K. Mikolajczyk, and J. Matas. Trackinglearning-detection. IEEE Trans. Pattern Anal. Mach. Intell., 34(7):1409-1422, July 2012.

[15] J. Kim, J. He, K. Lyons, and T. Starner. The gesture watch: A wireless contact-free gesture based wrist interface. In Proceedings of the 2007 11th IEEE International Symposium on Wearable Computers, ISWC '07, pages 1-8, Washington, DC, USA, 2007. IEEE Computer Society.

[16] S. W. Kim and S. Marti. Ghost fingers: a hybrid approach to the interaction with remote displays. In CHI' 12 Extended Abstracts on Human Factors in Computing Systems, CHI EA '12, pages 1877-1882, New York, NY, USA, 2012. ACM.

[17] Z. Lv and et al. Finger in air: Touch-less interaction on smartphone. In Proceedings of the 12st ACM International Conference on Mobile and Ubiquitous Multimedia, MUM '13, Luleå, Sweden, 2013. ACM.

[18] Z. Lv, M. S. L. Khan, and S. Réhman. Hand and foot gesture interaction for handheld devices. In Proceedings of the 21st ACM International Conference on Multimedia, MM '13, Barcelona, Catalunya, Spain, 2013. ACM.

[19] P. Mistry and P. Maes. Sixthsense: a wearable gestural interface. In ACM SIGGRAPH ASIA 2009 Sketches, SIGGRAPH ASIA '09, pages 11:1-11:1, New York, NY, USA, 2009. ACM.

[20] P. Mistry and P. Maes. Mouseless: a computer mouse as small as invisible. In CHI' 11 Extended Abstracts on Human Factors in Computing Systems, CHI EA '11, pages 10991104, New York, NY, USA, 2011. ACM.

[21] P. Mistry, P. Maes, and L. Chang. Wuw - wear ur world: a wearable gestural interface. In $\mathrm{CHI}$ '09 Extended $\mathrm{Ab}$ stracts on Human Factors in Computing Systems, CHI EA '09, pages 4111-4116, New York, NY, USA, 2009. ACM.

[22] A. Mittal, A. Zisserman, and P. H. S. Torr. Hand detection using multiple proposals. In British Machine Vision Conference, 2011.

[23] S. Réhman, A. Khan, and H. Li. Interactive Feet for Mobile Immersive Interaction. In ACM Int. Workshop MobiVis Workshop at MobileHCI, 2012.

[24] J. Rico and S. Brewster. Usable gestures for mobile interfaces: evaluating social acceptability. In Proceedings of the SIGCHI Conference on Human Factors in Computing Systems, CHI '10, pages 887-896, New York, NY, USA, 2010. ACM.

[25] N. Sangsuriyachot and M. Sugimoto. Novel Interaction Techniques based on a Combination of Hand and Foot Gestures in Tabletop Environments. In 10th Asia Pacific Conf. HCI, 2012.

[26] N. Ukita and M. Kidode. Wearable virtual tablet: fingertip drawing on a portable plane-object using an active-infrared camera. In Proceedings of the 9th international conference on Intelligent user interfaces, IUI '04, pages 169-176, New York, NY, USA, 2004. ACM.

[27] D. Valkov, F. Steinicke, G. Bruder, and K. Hinrichs. Traveling in 3D Virtual Environments with Foot Gestures and a Multi-Touch enabled WIM. In Int. Conf. Virtual Reality, 2010.
[28] J. Wang, S. Zhai, and J. Canny. Camera phone based motion sensing: interaction techniques, applications and performance study. In Proceedings of the 19th annual ACM symposium on User interface software and technology, UIST '06, pages 101-110, New York, NY, USA, 2006. ACM.

[29] D. Wigdor, C. Forlines, P. Baudisch, J. Barnwell, and C. Shen. Lucid touch: a see-through mobile device. In Proceedings of the 20th annual ACM symposium on User interface software and technology, UIST '07, pages 269-278, New York, NY, USA, 2007. ACM.

[30] S. Yousefi, F. Abedan Kondori, and H. Li. Experiencing real $3 \mathrm{~d}$ gestural interaction with mobile devices. Pattern Recogn. Lett., 34(8):912-921, June 2013. 